\title{
The effects of lockdown-induced air quality changes on the results of cardiac functional stress testing in coronary artery disease and heart failure patients
}

\author{
Antonello D'Andrea ${ }^{1} \cdot$ Quirino Ciampi $^{2} \cdot$ Antonello Russo $^{3}$ - Alberto Forni $^{1} \cdot$ Cristina Mangia $^{4} \cdot$ Eugenio Picano $^{5}$ (D) $\cdot$ on \\ behalf of the Stress Echo 2020 study group of the Italian Society of Echocardiography and Cardiovascular Imaging
}

Received: 20 January 2021 / Accepted: 18 March 2021 / Published online: 30 March 2021

(C) The Author(s), under exclusive licence to Springer-Verlag GmbH Germany, part of Springer Nature 2021

\begin{abstract}
In vulnerable subjects, the increase in air pollution worsens the signs of myocardial ischemia. Lockdown during COVID-19 pandemics substantially cleaned the air. The objective of this is to assess the effects of air cleaning due to lockdown on stress echocardiography (SE) results. We enrolled 19 patients with chronic coronary artery disease and/or heart failure referred to SE (semi-supine bicycle exercise, $n=8$, or dipyridamole, $n=11$ ). Before and soon after lockdown, we assessed regional wall motion abnormalities (abnormal value: worsening of $\geq 2$ segments), B-lines (a sign of pulmonary congestion, 4-site simplified scan, abnormal value $\geq 2$ ), and coronary flow velocity reserve in left anterior descending artery (CFVR, abnormal value $<2.0)$. Local air quality indicators (same day of SE) of fine particulate matter $\left(\mathrm{PM}_{2.5}\right)$ and nitrogen dioxide $\left(\mathrm{NO}_{2}\right)$ were obtained from publicly available data sets of the regional authority of environmental protection. After lockdown, $\mathrm{NO}_{2}$ concentration decreased from $19 \pm 10$ to $10 \pm 4 \mu \mathrm{g} / \mathrm{m}^{3}(p=0.006)$. After lockdown, abnormal responses remained unchanged for ischemia $(21 \% \mathrm{vs} 16 \%, p=\mathrm{ns})$ and decreased for B-lines $(42 \% \mathrm{vs} 5 \%, p=0.008)$ and CFVR ( 84 vs $42 \%, p=0.007)$. Changes in coronary flow velocity reserve (CFVR) were correlated to same-day variations in $\mathrm{NO}_{2}(r=$ $-0.578, p=0.010)$ and preceding 30-day changes in $\mathrm{PM}_{2.5}(r=-0.518, p=0.023)$. After lockdown, air cleaning was associated with a beneficial effect on coronary small vessel dysfunction and alveolar-capillary barrier distress mirrored by improvement of CFVR and Blines during SE in vulnerable patients. ClinicalTrials.gov Identifier: NCT 030.49995
\end{abstract}

Keywords Air pollution $\cdot$ Coronary artery disease $\cdot$ Heart failure $\cdot$ Stress echocardiography

\section{Introduction}

Air pollution contributes substantially to cardiovascular morbidity and mortality with about 8 million of all-cause avoidable deaths annually, a burden comparable to tobacco smoking (Lelieveld et al. 2019). Acute worsening of air quality increases the signs of exercise-induced myocardial ischemia in vulnerable subjects with pre-existing clinically overt

\begin{tabular}{|c|c|}
\hline Res & ponsible Editor: Lotfi Aleya \\
\hline$\triangle$ & $\begin{array}{l}\text { Eugenio Picano } \\
\text { picano@ifc.cnr.it }\end{array}$ \\
\hline & $\begin{array}{l}\text { Antonello D'Andrea } \\
\text { antonellodandrea@libero.it }\end{array}$ \\
\hline & $\begin{array}{l}\text { Quirino Ciampi } \\
\text { qciampi@gmail.com }\end{array}$ \\
\hline & $\begin{array}{l}\text { Antonello Russo } \\
\text { antonellorusso72@ hotmail.com }\end{array}$ \\
\hline & $\begin{array}{l}\text { Alberto Forni } \\
\text { alberto.forni@gmail.com }\end{array}$ \\
\hline
\end{tabular}

Cristina Mangia

c.mangia@isac.cnr.it

1 Cardiology Division, Nocera Inferiore Hospital, Nocera Inferiore, Italy

2 Cardiology Division, Fatebenefratelli Hospital, Benevento, Italy

3 Association for Public Health "Salute Pubblica", Brindisi, Italy

4 ISAC-Institute of Sciences of Atmosphere and Climate, CNR, Lecce, Italy

5 Institute of Clinical Physiology, Biomedicine Department, CNR, Pisa, Italy 
disease and abnormal response to cardiac functional testing (Allred et al. 1989; Pekkanen et al. 2002). The increased concentration of air pollutants may affect cardiovascular function favoring the induction of myocardial ischemia with complex molecular and cellular mechanisms characterized by increased inflammatory and oxyradical stress potentially impairing endothelial, smooth muscle cell, myocardial, alveolar, and neuronal function (Brook et al. 2010; Newby et al. 2015; Rajagopalan et al. 2018). With state-of-the-art protocols, exercise or vasodilator stress echo (SE) allows a comprehensive evaluation of inducible ischemia, pulmonary congestion, cardiac reserve, coronary flow velocity reserve, and cardiac autonomic balance (Picano et al. 2018).

Simple studies made on the basis of clinical observation can be a source of extraordinary information when performed under extraordinary circumstances. The air cleaning achieved with lockdown was unprecedented and opened a window of opportunity to study the incompletely understood links between air pollution and cardiac vulnerability (Picano 2020). The current study hypothesis was that lockdown-induced drop-off in air pollution affects SE results. To test this hypothesis, the pre- and post-lockdown ABCDE-SE and local air quality data from regional environmental protection agency were evaluated in 19 patients with coronary artery disease (CAD) and/or heart failure (HF) referred to clinically driven SE in the ongoing Stress Echo 2020 multicenter study (Picano and Ciampi 2017).

\section{Methods}

\section{Study population}

We initially screened 24 patients with known or suspected $\mathrm{CAD}$ and/or $\mathrm{HF}$ recruited by $2 \mathrm{SE}$ laboratories (Nocera Inferiore and Benevento, Campania Region, Italy). Lockdown started on March 9th, 2020, and was lifted on May 4th, 2020. The inclusion criteria were (1) previous maximal physical or pharmacological SE performed in the 12 months before lockdown, with abnormal result of at least one criterion; (2) sinus rhythm (necessary to have standardized criteria for heart rate reserve); (3) follow-up SE in the 3 weeks after lockdown release at reopening of outpatient activity; and (4) willingness to give their written informed consent allowing scientific utilization of observational data, respectful of privacy rights.

From the initial set of 24 , we excluded 5 patients for one of the following exclusion criteria: (1) change in clinical status with acute events between the 2 examinations $(n=2)$; (2) change in pharmacological therapy by referring physician with introduction of new drugs absent at initial evaluation ( $n$ $=2$ ); and (3) occurrence of atrial fibrillation between the 2 tests $(n=1)$.
All patients underwent SE with either semi-supine bicycle exercise $(n=8)$ or high dose dipyridamole $(n=11)$ according to the recommendations of international scientific societies (Pellikka et al. 2020). In patient capable to exercise, semisupine bicycle was the stress modality of choice. Imaging was performed at baseline, at an initial workload of 25 watts, at each stage, at peak stress, and in recovery. The workload was increased at increments of 25 watts every 2 min. In patients unable to exercise adequately or with contraindications to exercise, dipyridamole $(0.84 \mathrm{mg} / \mathrm{kg}$ over 6 ') was used (Pellikka et al. 2020; Sicari et al. 2008).

In each patient, the same test was employed for the first and the second examination. Written informed consent was obtained from all patients before testing. The study protocol was reviewed and approved by the institutional ethics committees as a part of SE 2020 study (148-Comitato Etico Lazio1, July 16, 2016; ClinicalTrials.gov Identifier: NCT 030. 49995). The analysis of correlation with air quality data was not a prespecified analysis and did not interfere with data collection or clinical management (amendment approved 1424/2020 CE Lazio). The study was funded partly by the Italian National Research Council (Ageing project, P001328) and with travel grants of the Italian Society of Echocardiography and Cardiovascular Imaging with dedicated sessions during national meetings. No support from industry was received.

\section{Stress echocardiography}

We used commercially available ultrasound machines. All patients underwent comprehensive transthoracic echocardiography (TTE) at rest. Measurements were performed according to the recommendations of the American Society of Echocardiography and European Association of Cardiovascular Imaging (Lang et al. 2015).

\section{$A B C D E$ protocol}

The ABCDE protocol of SE was used when each laboratory had completed the upstream quality control process as previously detailed (Ciampi et al. 2019). Echocardiographic imaging was performed from parasternal long axis view, short axis view, and apical 4-, 3-, and 2-chamber view, using conventional 2-dimensional echocardiography (Pellikka et al. 2020). Step A included assessment of wall motion abnormalities and was performed in all patients. Wall motion score index (WMSI) was calculated in each patient at baseline and peak stress, in a four-point score ranging from 1 (normal) to 4 (dyskinetic) in a 17-segment model of the left ventricle. The A criterion was considered positive in presence of stress WMSI > rest WMSI in at least 2 segments (Ciampi et al. 2019). Step B included the assessment of B-lines with lung ultrasound. We adopted the 4-site simplified scan (Scali et al. 
2020). We analyzed the anterior and lateral hemithorax, scanning from midaxillary to mid-clavicular lines on the third intercostal space. A total of 4 chest sites was scanned, and each site had a score from 0 to 10 . The $\mathrm{B}$ criterion was considered positive in presence of stress B-lines $\geq 2$ units (Scali et al. 2020). Step $C$ of protocol included the force-based assessment of left ventricular contractile reserve as the stress/rest ratio of force, calculated as systolic blood pressure/end-systolic volume. The $\mathrm{C}$ criterion was considered abnormal in presence of left ventricular contractile reserve $\leq 1.1$ for dipyridamole or $\leq$ 2.0 for exercise (Ciampi et al. 2019). Coronary flow velocity reserve (step D) was assessed in mid-distal left anterior descending artery during the standard SE examination using intermittent imaging of wall motion and flow (Sicari et al. 2008). The D criterion was considered abnormal in presence of coronary flow velocity reserve $\leq 2.0$ for both dipyridamole and exercise (Ciampi et al. 2019). The last imagingindependent step of the ABCDE protocol was the E step, based on EKG. Heart rate reserve (step E) was calculated as the peak/rest HR from 12-lead EKG. A blunted heart rate reserve is a prognostically relevant index of reduced sympathetic reserve and abnormal cardiac autonomic function. The $\mathrm{E}$ criterion was considered abnormal in presence of heart rate reserve $\leq 1.22$ for dipyridamole or $\leq 1.80$ for exercise (Ciampi et al. 2019). The procedure for acquisition between centers was standardized through a web-based learning module before starting data collection. Assessors (ADA and QC) were blinded to the study condition and air quality data. Inter- and intra-observer reproducibility was $>90 \%$ for all tested SE parameters as previously shown (Ciampi et al. 2019).

\section{Air quality data}

Local air quality data were obtained from publicly available data sets from regional authority of environmental protection. For each patient and each test of the same patient, the values of 2 particulate and 5 gaseous pollutants were collected when available: fine particulate matter with aerodynamic diameter $<2.5 \mu \mathrm{m}\left(\mathrm{PM}_{2.5}\right)$, particulate matter with aerodynamic diameter $<10 \mu \mathrm{m}\left(\mathrm{PM}_{10}\right), \mathrm{NO}_{2}$, ozone, carbon monoxide, sulfur dioxide, and benzene (Environmental Protection Agency 2014). Values of the same day of testing were taken as representative of that specific condition using the air monitor point closest to the patient house (on average within few kilometers). When the values were not available for malfunction or other reasons, the previous day values were considered. Air quality data were collected and inputted by an assessor (AR) unaware of the patient identity, condition, and functional test findings. The same-day values and the average of 3-, 5-, and 30-day before testing were analyzed. Values of humidity (\%), temperature $\left({ }^{\circ} \mathrm{C}\right)$ and atmospheric pressure were also collected from Centro Meteorologico e Climatologico (CEMEC, Meteorologic and Climatologic Center) of Arpa Campania http://cemec.arpacampania.it/meteoambientecampania/php/ misure_suolo.php.

\section{Statistical analysis}

Categorical data are expressed in terms of number of subjects and percentage while continuous data are expressed as mean \pm standard deviation or median (minimum-maximum) depending on variables' distribution. For continuous variables intergroup differences were tested with Student's $t$-test for paired values. Ordinary least squares regression was used to assess the correlation between functional test and air quality data. Statistical significance was set at $p<0.05$. All analyses were performed using STATA (STATACorp. Stata statistical software: Release 14. College Station, TX: STATACorp LP) and $\mathrm{R}$ version 3.6.

\section{Results}

The patients' characteristics are reported in Table 1. The average interval between the first and the second test was $329 \pm$ 97 days (range 116-402). The second test was performed $12 \pm$ 4 days after the end of lockdown (range 7-21 days).

\section{SE findings}

No patient developed significant adverse events or limiting side effects during testing. After lockdown, positivity rate at study entry decreased for steps $\mathrm{B}$ and $\mathrm{D}$ and remained unchanged for step A, C, and E (Fig. 1).

Table 1 Study population

\begin{tabular}{lc}
\hline Variable & $\begin{array}{c}\text { Overall population } \\
(N=19)\end{array}$ \\
\hline Male/female sex, $n(\%)$ & $13 / 5(68 / 32 \%)$ \\
Age, years & $63 \pm 6$ \\
BMI, kg/m ${ }^{2}$ & $2.02 \pm 0.17$ \\
CAD/HF & $13 / 6$ \\
Previous PCI & $9(47 \%)$ \\
Hypertension, $n(\%)$ & $13(68 \%)$ \\
Diabetes mellitus, $n(\%)$ & $9(47 \%)$ \\
Smoking, $n(\%)$ & $8(42 \%)$ \\
Ejection fraction, $\%$ & $48 \pm 7$ \\
Beta-blockers, $N(\%)$ & $17(89 \%)$ \\
ACE inhibitors or sartans, $N(\%)$ & $16(84 \%)$ \\
Nitrates, $N(\%)$ & $3(16 \%)$ \\
Diuretics, $N(\%)$ & $6(32 \%)$ \\
\hline
\end{tabular}

$A C E$, angiotensin-converting enzyme; $B M I$, body mass index; $C A D$, coronary artery disease; $H F$, heart failure; $M R$, mitral regurgitation 


\section{Air quality findings}

Concentration of $\mathrm{PM}_{2.5}(-40 \%)$ and $\mathrm{PM}_{10}(-15 \%)$ did not change significantly (Fig. 2). There was a significant reduction in $\mathrm{NO}_{2}(-47 \%)$, sulfur dioxide $(-78 \%)$, benzene $(-60 \%)$, and carbon monoxide $(-55 \%)$ after lockdown indicating an improvement of air quality. Humidity tended to decrease $(59 \pm$ 24 vs $51 \pm 7 \%, p=0.07$ ), temperature increased ( $13 \pm 5$ vs 20 $\left.\pm 3{ }^{\circ} \mathrm{C}, p<0.001\right)$, and atmospheric pressure remained unchanged ( $1011 \pm 4$ vs $1010 \pm 4$ millibar, $p=0.23)$ in the postlockdown assessment.

\section{Correlation between air quality and stress echo results}

Coronary flow velocity reserve changes were correlated with same-day changes in $\mathrm{NO}_{2}$ (Fig. 3) and benzene $(r=-584, p=$ $0.014, n=17)$. For $\mathrm{NO}_{2}$, similar results were observed if we analyzed the same day of testing and the average of 3 days $(r=$ $-0.584, p=0.014), 5$ days $(r=-0.550, p=0.015)$, and 30 days $(r=-0.249, p=0.014)$ before SE. For benzene, the statistical significance was lost if we analyzed the average of 3 days $(r=-338, p=0.17, n=18), 5$ days $(r=-456, p=$ $0.057, n=18)$, and 30 days $(r=-319, p=0.198, n=18)$ prior to $\mathrm{SE}$. For $\mathrm{PM}_{2.5}$, there was a significant negative correlation with changes in coronary flow velocity reserve considering the average of 5 days $(r=-0.486, p=0.035)$, and 30 days ( $r=-0.518, p=0.023$ ) before $\mathrm{SE}$, but the statistical significance was lost if we analyzed the average of same day $(r=$ $-449, p=0.62, n=18)$, and 3 days $(r=-389, p=0.10, n=19)$ prior to SE. For $\mathrm{PM}_{10}$, there was a significant negative correlation with changes in coronary flow velocity reserve considering the average of 30 days ( $r=-0.563, p=0.012$ ) before SE but the statistical significance was lost if we analyzed the average of same day $(r=-304, p=0.22, n=18), 3$ days $(r$ $=-254, p=0.29, n=19)$, or 5 days $(r=-345, p=0.14, n=$ 19) prior to $\mathrm{SE}$.

Changes in B-lines were not correlated with any specific pollutant at any time point (same day, 3 days, 5 days, 30 days).

\section{Discussion}

In the first weeks after the lift of lockdown the air quality improved when compared to pre-lockdown days, mainly for a marked reduction (between 47 and 78\%) in $\mathrm{NO}_{2}$, sulfur dioxide, benzene, and carbon monoxide accompanied by a non-significant decrease in fine particulate matter $(-40 \%)$. Air cleaning was associated with neutral effects on inducible ischemia, LV contractile function, and cardiac autonomic balance, but showed beneficial effects on coronary microvascular function and pulmonary congestion. The available data on
Fig. 1 ABCDE stress echo results before and after lockdown. The percentage of positivity decreased for steps B and D and remained unchanged for steps $\mathrm{A}, \mathrm{C}$, and $\mathrm{E}$. Dashed column: before lockdown. Empty column: after lockdown

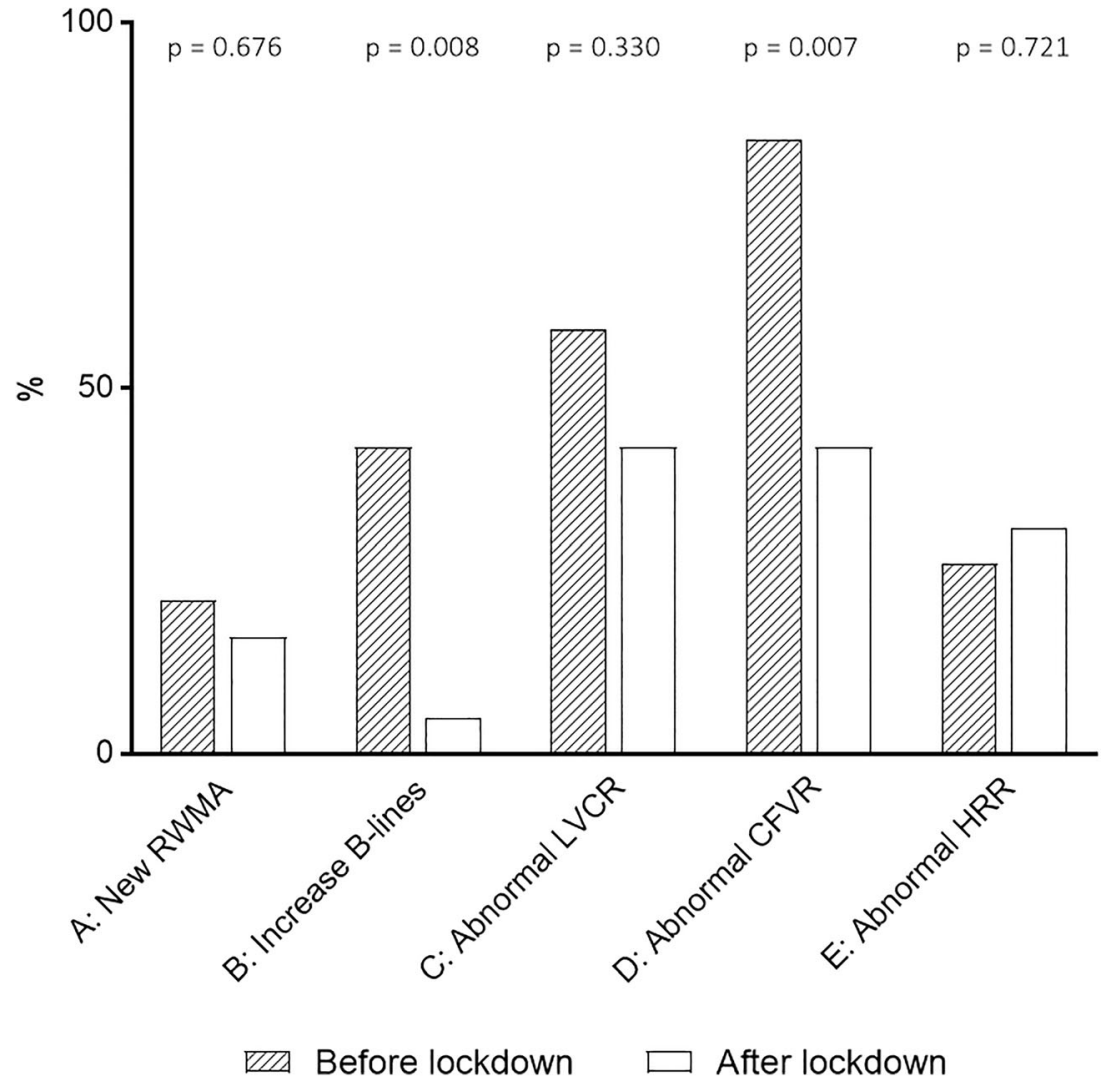


Fig. 2 Air pollutants results before and after lockdown. The concentration of air pollutants remained unchanged for $\mathrm{PM}_{10}$ and $\mathrm{PM}_{2.5}$, markedly decreased for $\mathrm{NO}_{2}$ and sulfur dioxide, and increased for ozone. Dashed column: before lockdown. Empty column: after lockdown

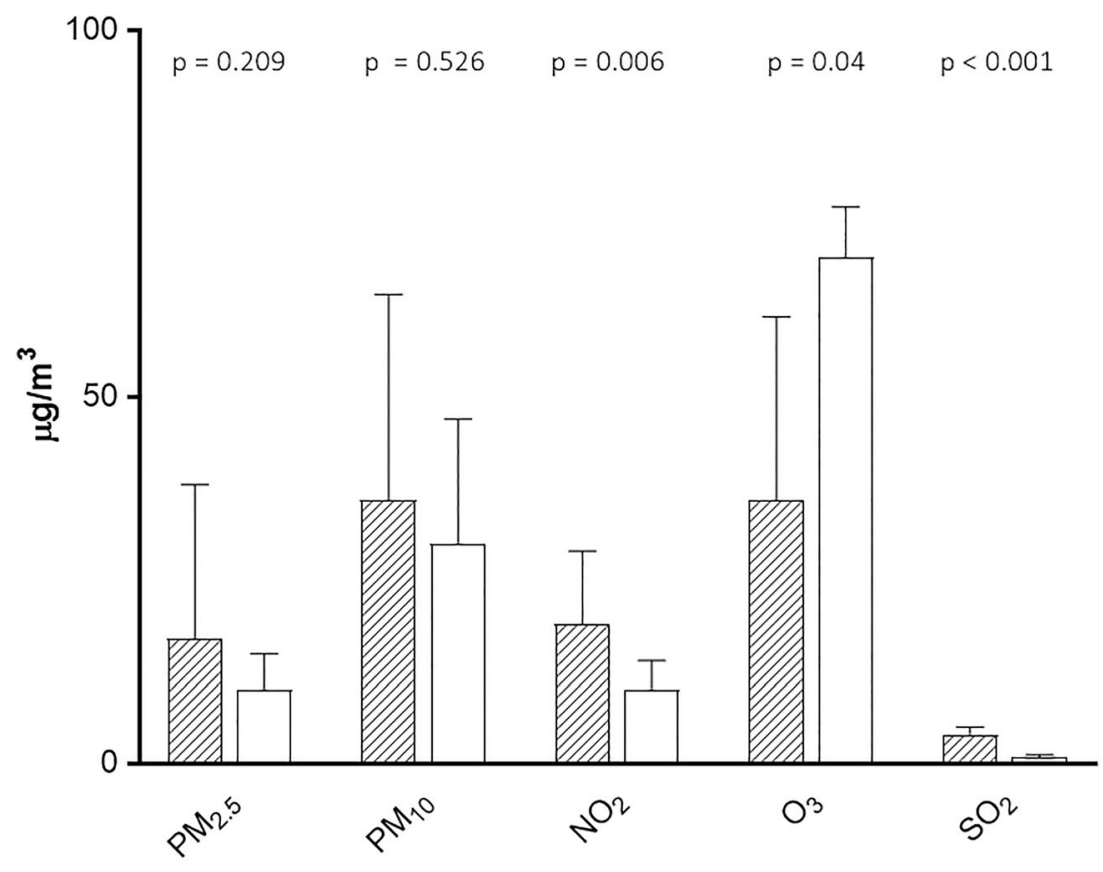

एय Before lockdown $\square$ After lockdown cardiovascular effects of air pollution are mostly focused on fine particulate matter but changes in $\mathrm{NO}_{2}$, benzene, and carbon monoxide may also account for the observed variations. $\mathrm{NO}_{2}$ exerts a detrimental cardiovascular effect through augmented inflammatory and oxyradical stress at the lung, heart, and systemic level. Increased inflammatory stress acts either directly or indirectly through specific molecules such as nitric oxide to impair coronary blood vessel vasodilation reducing coronary flow velocity reserve (Münzel et al. 2018). The increased production of inflammatory cytokines by leukocytes alters the alveolar-capillary barrier increasing its permeability to water filtration into the lung extravascular space for any given intravascular pressure (Chiu et al. 2019). By selection, patients were clinically stable without obvious changes in anginal class or dyspnea class. SE changes were therefore observed at a sub-clinical level, without a clear modification in
Fig. 3 The correlation between percent $\mathrm{NO}_{2}$ and percent coronary flow velocity reserve changes before and after lockdown. $\mathrm{x}$ axis: changes in coronary flow velocity reserve; y-axis: changes in $\mathrm{NO}_{2}$ after lockdown

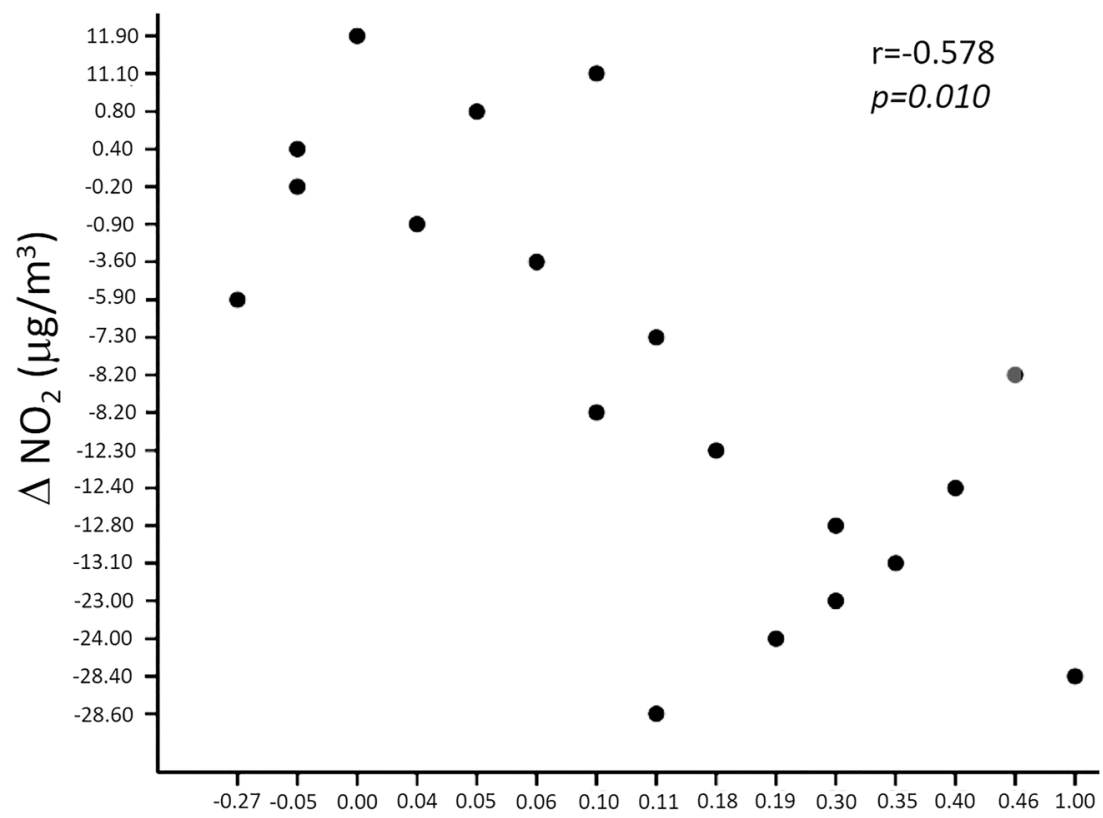

$\triangle$ CFVR 
clinical status or in medication or lifestyle compliance. Nevertheless, the magnitude of observed changes can be clinically meaningful since for instance the average improvement of CFVR from 1.8 to 2.03 means that some patients shifted from an "abnormal" to a "normal" result. More realistically, the levels of risk associated with CFVR are continuous rather than binary, and the lower the CFVR the higher the risk even for normal or abnormal values (Ciampi et al. 2019).

\section{Comparison with previous studies}

Air cleaning may exert beneficial effects on results of stress testing. In patients with $\mathrm{CAD}$, the increase in $\mathrm{PM}_{2.5}, \mathrm{NO}_{2}$, and carbon monoxide in the days or hours before testing is associated to greater signs of ischemia at Holter monitoring and during exercise testing (Aronow et al. 1972; Anderson et al. 1973; Adams et al. 1988; Kleinman et al. 1989).

The rise of ozone during lockdown can be due to seasonal changes with temperature rise and is consistent with previous studies on air quality worldwide (Bauwens et al. 2020). Ozone at ground level is a secondary pollutant destroyed by nitrogen dioxide, so that ozone levels can increase when $\mathrm{NO}_{2}$ concentration falls. However, ozone on its turn has complex cardiovascular detrimental effects, including stimulation of sympathetic activity and arterial vasoconstriction (Hadley et al. 2018). This may contribute to explaining the absence of net effects on inducible ischemia, contractile reserve, and heart rate reserve which one could expect from a reduction in carbon monoxide, $\mathrm{NO}_{2}$, and fine particulate matter. Not all pollutants change in the same direction, and some changes may have antagonistic effects on cardiovascular parameters.

\section{Clinical implications}

Air pollution changes affect results of functional testing and should be considered in the evaluation of test results. To put the results of functional testing in context, cardiologists should be aware of the concentration of air pollutants to which the patient is exposed every day outdoor, at work, and in house. Risk stratification and phenotyping of disease are based upon response to specific variables and at least some of them such as CFVR and B-lines can be affected by changes in air pollution falling well within the range of acceptable values. The recognition of this variable is especially important since air pollution can be considered today an actionable therapeutic target, for instance with air cleaners and personal protection devices such as face masks (Al-Kindi et al. 2020).

\section{Study limitations}

The major limitation of the study is the small sample size. This was due to the limited number of patients arriving to stress testing in the early post-lockdown days, when outpatient imaging activity in cardiology was dramatically reduced. Furthermore, only patients with previous (prelockdown) testing could be included in the present study. In addition, numbers are too small to allow a statistically plausible separate analysis of CAD and HF groups.

The study is observational, with all potential confounders of a non-randomized design.

Air quality cannot be characterized by a single parameter, and each component of the complex mixture of particles, gasses, and liquids contributing to air quality can have independent effects.

The observational study design did not allow a crossover, and the observed changes could be attributed to variations in the natural history of the disease. However, spontaneous improvements are unlikely in patients with chronic but progressive disease such as CAD or HF. Air quality assessors were blinded to stress imaging results and vice versa. We used variations in the same patient as the meaningful indicator, which may average out many potential confounders.

This is a pilot study and now a systematic evaluation of air pollutants and stress testing results is planned in 600 patients recruited by $20+$ centers from $15+$ countries in the Stress Echo 2030 study, just started, in the subproject ESTER (Environmental Stress echocardiography and Air Pollution). The study will include CAD and/or HF patients but also patients with hypertrophic cardiomyopathy, valvular heart disease, and patients post-COVID-19, since air pollution may potentially affect all aspects of cardiac functional testing in virtually all types of patients.

\section{Conclusion}

In patients with chronic $\mathrm{CAD}$ and/or $\mathrm{HF}$, the improvement in air quality well within the European Union air quality standards is associated with an improvement of cardiac stress test results related to coronary microvascular function and pulmonary congestion. Changes in air pollution did not affect imaging biomarkers of inducible ischemia, contractile reserve, or cardiac autonomic function. Air quality data are available in real time with freely downloadable apps, and they should probably be considered in the evaluation of cardiac functional test results.

Abbreviations $\mathrm{CAD}$, Coronary artery disease; EF, Ejection fraction; $\mathrm{HF}$, Heart failure; $\mathrm{NO}_{2}$, Nitrogen dioxide; $\mathrm{PM}_{2.5}$, Fine particulate matter with aerodynamic diameter $<2.5 \mu \mathrm{m} ; \mathrm{PM}_{10}$, Particulate matter with aerodynamic diameter $<10 \mu \mathrm{m}$; RWMA, Regional wall motion abnormalities; SE, Stress echocardiography; TTE, Transthoracic echocardiography; WMSI, Wall motion score index

Acknowledgements The technical support of Michele De Nes is gratefully acknowledged. He is computer scientist of the Italian National Research Council-Institute of Clinical Physiology. 
Author contribution $\mathrm{ADA}$ and $\mathrm{AF}$ recruited 14 patients, contributed to draft the first version of the manuscript, and approved the submitted version; $\mathrm{AR}$ and $\mathrm{CM}$ are the environmental epidemiologists responsible for collection, analysis, and evaluation of air quality data; QC recruited 5 patients, contributed to data analysis, and approved the submitted version; and EP had the original idea, is the study chairman, designed the protocol, organized the content of web-based training, contributed to data interpretation, and drafted the manuscript.

Funding The study was funded partly by the Italian National Research Council (Ageing project, P001328) and with travel grants of the Italian Society of Echocardiography and Cardiovascular Imaging with dedicated sessions during national meetings. No support from industry was received.

Data availability Supporting data set will be made available upon reasonable request.

\section{Declarations}

Ethics approval The study protocol was reviewed and approved by the institutional ethics committees as a part of SE 2020 study (148-Comitato Etico Lazio-1, July 16, 2016; ClinicalTrials.gov Identifier: NCT 030. 49995). The analysis of correlation with air quality data was not a prespecified analysis and did not interfere with data collection or clinical management (amendment approved 1424/2020 CE Lazio).

Consent to participate All the patients provided their informed consent for participation to the study.

Consent for publication All the authors have read and approved the manuscript and accorded the consent for publication.

Competing interests The authors declare no competing interests.

\section{References}

Adams KF, Koch G, Chatterjee B, Goldstein GM, O'Neil JJ, Bromberg PA, Sheps DS (1988 Oct) Acute elevation of blood carboxyhemoglobin to $6 \%$ impairs exercise performance and aggravates symptoms in patients with ischemic heart disease. J Am Coll Cardiol 12(4):900-909. https://doi.org/10.1016/0735-1097(88) 90452-4

Al-Kindi SG, Brook RD, Biswal S, Rajagopalan S (2020) Environmental determinants of cardiovascular disease: lessons learned from air pollution. Nat Rev Cardiol 17(10):656-672. https://doi.org/10.1038/ s41569-020-0371-2

Allred EN, Bleecker ER, Chaitman BR, Dahms TE, Gottlieb SO, Hackney JD, Pagano M, Selvester RH, Walden SM, Warren J (1989 Nov 23) Short-term effects of carbon monoxide exposure on the exercise performance of subjects with coronary artery disease. N Engl J Med 321(21):1426-1432. https://doi.org/10.1056/ NEJM198911233212102

Anderson EW, Andelman RJ, Strauch JM, Fortuin NJ, Knelson JH (1973 Jul) Effect of low-level carbon monoxide exposure on onset and duration of angina pectoris. A study in ten patients with ischemic heart disease. Ann Intern Med 79(1):46-50. https://doi.org/10.7326/ 0003-4819-79-1-46

Aronow WS, Harris CN, Isbell MW, Rokaw SN, Imparato B (1972 Nov) Effect of freeway travel on angina pectoris. Ann Intern Med 77(5): 669-676. https://doi.org/10.7326/0003-4819-77-5-669
Bauwens M, Compernolle S, Syavrakou T et al (2020) Impact of coronavirus outbreak on $\mathrm{NO}_{2}$ pollution assessed using TROPOMI and OMI observations. Geophys Res Lett. https://doi.org/10.1029/ 2020GL087978

Brook RD, Rajagopalan S, Pope CA 3rd et al (2010) Particulate matter air pollution and cardiovascular disease: an update to the scientific statement from the American Heart Association. Circulation. 121: 2331-2378

Chiu PF, Chang CH, Wu CL et al (2019) High particulate matter 2.5 levels and ambient temperature are associated with acute lung edema in patients with nondialysis stage 5 chronic kidney disease. Nephrol Dial Transplant 34:1354-1360

Ciampi Q, Zagatina A, Cortigiani L et al (2019) Functional, coronary anatomic and prognostic correlates of coronary flow velocity reserve during stress echocardiography. J Am Coll Cardiol 74:2280-2293

Environmental Protection Agency (2014) Air quality index. A guide to air quality and your health. https://www3.epa.gov/airnow/aqi brochure 02 14.pdf

Hadley MB, Vedanthan R, Fuster V (2018) Air pollution and cardiovascular disease: a window of opportunity. Nat Rev Cardiol 15:193194

Kleinman MT, Davidson DM, Vandagriff RB, Caiozzo VJ, Whittenberger JL (1989 Nov-Dec) Effects of short-term exposure to carbon monoxide in subjects with coronary artery disease. Arch Environ Health 44(6):361-369. https://doi.org/10.1080/00039896. 1989.9935908

Lang RM, Badano LP, Mor-Avi V, Afilalo J, Armstrong A, Ernande L, Flachskampf FA, Foster E, Goldstein SA, Kuznetsova T, Lancellotti P, Muraru D, Picard MH, Rietzschel ER, Rudski L, Spencer KT, Tsang W, Voigt JU (2015) Recommendations for cardiac chamber quantification by echocardiography in adults: an update from the American Society of Echocardiography and the European Association of Cardiovascular Imaging. J Am Soc Echocardiogr 28:1-39

Lelieveld J, Klingmüller K, Pozzer A, Pöschl U, Fnais M, Daiber A, Münzel T (2019) Cardiovascular disease burden from ambient air pollution in Europe reassessed using novel hazard ratio functions. Eur Heart J 40(20):1590-1596. https://doi.org/10.1093/eurheartj/ ehz135

Münzel T, Gori T, Al-Kindi S et al (2018) Effects of gaseous and solid constituents of air pollution on endothelial function. Eur Heart J 39: 3543-3550

Newby DE, Mannucci PM, Tell GS, Baccarelli AA, Brook RD, Donaldson K, Forastiere F, Franchini M, Franco OH, Graham I, Hoek G, Hoffmann B, Hoylaerts MF, Künzli N, Mills N, Pekkanen J, Peters A, Piepoli MF, Rajagopalan S, Storey RF, ESC Working Group on Thrombosis, European Association for Cardiovascular Prevention and Rehabilitation, ESC Heart Failure Association (2015) Expert position paper on air pollution and cardiovascular disease. Eur Heart J 36:83-93

Pekkanen J, Peters A, Hoek G, Tiittanen P, Brunekreef B, de Hartog J, Heinrich J, Ibald-Mulli A, Kreyling WG, Lanki T, Timonen KL, Vanninen E (2002) Particulate air pollution and risk of STsegment depression during repeated submaximal exercise tests among subjects with coronary heart disease: the Exposure and Risk Assessment for Fine and Ultrafine Particles in Ambient Air (ULTRA) study. Circulation. 106:933-938

Pellikka PA, Arruda-Olson A, Chaudhry FA et al (2020) Guidelines for performance, interpretation, and application of stress echocardiography in ischemic heart disease: from the American Society of Echocardiography. J Am Soc Echocardiogr 33(1):1-41.e8. https:// doi.org/10.1016/j.echo.2019.07.001

Picano E (2020) Where have all myocardial infarctions gone? The answer is blowing in the less polluted wind. Eur Heart J 41:2146-2147

Picano E, Ciampi Q (2017) Citro R, et al Stress echo 2020: The international stress echo study in ischemic and non-ischemic heart disease. 
Cardiovasc Ultrasound 15(1):3. https://doi.org/10.1186/s12947016-0092-1

Picano E, Ciampi Q, Wierzbowska-Drabik K, Urluescu M-L, Morrone D, Carpeggiani C (2018) The new clinical standard of integrated quadruple stress echocardiography with $\mathrm{ABCD}$ protocol. Cardiovasc Ultrasound 16(1):22. https://doi.org/10.1186/s12947-018-0141-z

Rajagopalan S, Al-Kindi SG, Brook RD (2018) Air pollution and cardiovascular disease: JACC state-of-the-art review. J Am Coll Cardiol 72:2054-2070

Scali MC, Ciampi Q, Zagatina A, Cortigiani L et al on behalf of the Stress Echo 2020 Study Group of the Italian Society of Echocardiography and Cardiovascular Imaging. Lung ultrasound and pulmonary congestion during stress echocardiography. J Am Coll Cardiol Img
13(August):2085-2095. https://doi.org/10.1016/j.jcmg.2020.04. 020

Sicari R, Nihoyannopoulos P, Evangelista A, Kasprzak J, Lancellotti P, Poldermans D, Voigt JU, Zamorano JL, on behalf of the European Association of Echocardiography (2008) on behalf of the European Association of Echocardiography. Stress echocardiography expert consensus statement. European Association of Echocardiography (EAE) (a registered branch of the ESC). Eur J Echocardiogr 9: $415-437$

Publisher's note Springer Nature remains neutral with regard to jurisdictional claims in published maps and institutional affiliations. 